\title{
Noval advance non-linear descriptor and characteristic equivalency to predict CT from MRI image
}

\author{
Sowmya Bachu, Shrutibhargava Choubey, Abhishek Choubey \\ Sreenidhi Institute of Science and Technology, India
}

\begin{tabular}{l} 
Article Info \\
\hline Article history: \\
Received Dec 28, 2019 \\
Revised Jan 12, 2020 \\
Accepted Feb 11, 2020 \\
\hline Keywords: \\
CT calculation \\
KNN regression \\
Low-rank approximation \\
Non-linear descriptor \\
PET attenuation correction
\end{tabular}

\begin{abstract}
Attenuation correction designed for PET/MR hybrid imaging frameworks along with portion making arrangements used for MR-based radiation treatment remain testing because of lacking high-energy photon weakening data. We present a new method so as to uses the learned nonlinear neighborhood descriptors also highlight coordinating toward foresee pseudoCT pictures starting T1w along with T2w MRI information. The nonlinear neighborhood descriptors are acquired through anticipating the direct descriptors interested in the nonlinear high-dimensional space utilizing an unequivocal constituent guide also low-position guess through regulated complex regularization. The nearby neighbors of every near descriptor inside the data MR pictures are looked during an obliged spatial extent of the MR pictures among the training dataset. By that point, the pseudo-CT patches are evaluated through k-closest neighbor relapse. The planned procedure designed for pseudo-CT forecast is quantitatively broke downward on top of a dataset comprising of coordinated mind MRI along with CT pictures on or after 13 subjects.
\end{abstract}

This is an open access article under the CC BY-SA license.

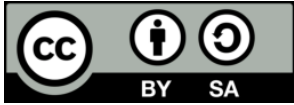

\section{Corresponding Author:}

Sowmya Bachu,

Sreenidhi Institute of Science and Technology,

Yamnampet, Ghatkesar, Hyderabad, Telangana 501301, India.

Email: Sb303107@gmail.com

\section{INTRODUCTION}

Numerous innovative methods contain be planned in favor of predicting CT imagery starting MRI data, as well as container, be classify interested in four classes: segmentation, atlas, exact series- also patchbased techniques. Inside the segmentation-based techniques [1-3], magnetic resonance imagery is segmented interested inside dissimilar hankie lessons (e.g., soft tissue, fat, and atmosphere, along with clean). Every group is next assigned the pre-defined reduction coefficients (LAC) otherwise computed tomography assessments. Toward get correct segmentation, the fuzzy clustering method along with SPM8 software were utilized inside [1, 2] also [3], correspondingly. The accurateness of the segmentation-based methods designed for pCT calculation be incomplete since the segmented tissue regions contribute in the direction of the similar pre-defined computed tomography assessment also the variation inside the proper computed tomography assessment surrounded through the similar hankie be unseen.

Basic fundamental design of the atlas-based techniques [4-6] exists straightforward. A dataset so as to contain numerous magnetic resonance/ computed tomography image pairs be necessary. Initial, an atlas dataset be register toward an input magnetic resonance imaging picture through calculating the deformation field among the atlas along with the magnetic resonance imaging picture. Next, the equivalent computed tomography imagery be distorted toward this MRI image by means of the multi-atlas information dissemination plan introduced within [5,6]. Finally, the obtained CT imagery is merged interested in the CT 
calculation. Within the picture synthesis stage, the Gaussian procedure weakening [5], limited picture comparison-based advance [5] also voxel-wise maximum likelihood strength averaging advances [6] be utilized also validate. The presentation of the atlas-based methods depends powerfully under top of the registration exactness along with the enduring populations encompass through the atlas. However, the atlas might fail to correspond toward the reduction of patients who contain a portion of their skulls detached.

Suitable toward the particularly towering reduction assessment of the skeleton, the exact calculation of clean inside predicted computed tomography is preferred. This require to facilitate the magnetic resonance picture container provide sufficient clue toward recognize the skeleton. Though, predictable magnetic resonance imaging signal be essentially inappropriate in favor of depicting bones as well as atmosphere structures due to the proton density dominance also the magnetic resonance imaging signal recreation mechanism. In recent times, numerous methods based on top of exact imaging sequences include are planned [7-12]. Ultra short echo time sequence (UTE) also zero echo time sequence (ZTE) was use toward improves the bone recognition inside [7-11] along with [12], correspondingly. The major constraint of this method is the further instance rate within progression information attainment inside the medical applications.

During this learning, a patch-based technique called characteristic corresponding through learned non-linear descriptors (FMLND) is planned on behalf of predicting pCT starting MRI records. Toward get better the ability of the clean recognition, a mixture of thick scale-invariant feature transforms (SIFT) descriptors by normalize unprocessed areas are used because the main descriptor of magnetic resonance imagery relatively than magnetic resonance raw patches otherwise voxels since here $[4,5,8,10,13,14,16]$. Scale Invariant Feature Transform characteristic depicts structural information, which is precious inside recognizing clean hankie along with atmosphere inside magnetic resonance imaging information. On the way to better hold the non-linearity of mapping among the main descriptors along with the computed tomography unprocessed areas, the main descriptors are predictable toward a high-dimensional gap by means of open feature maps [17] toward get widespread MRI information. When recommended within our preceding learn [18], the mapping starting the main descriptors toward the computed tomography unprocessed patches container exist roughly measured because nearby linear below the nearby spatial limitation. A supervised learning technique is planned on behalf of ensure the viability of the overstatement through learn a local nonlinear descriptor (LND), which be a widespread low-rank estimate of non-linear descriptors. During this knowledge construction, the comparison information of the computed tomography areas is used on behalf of regularization along with supervises the dimensionality decrease of the learned non-linear descriptors.

\section{LITERATURE SURVEY}

Calculations of CT substitutes starting MR imagery are clinically preferred used in favor of dose preparation inside MR-based radiation therapy along with reduction alteration within PET/MR [13]. Allowing for that present is no worldwide relation among intensities within MR along with CT imagery, we suggest local sparse correspondence combination (LSCC) used in support of the calculation of CT substitute on or after MR imagery. During LSCC, we suppose to MR as well as CT patches are situated under top of two nonlinear manifolds along with the mapping on or after the magnetic resonance diverse toward the computed tomography diverse approximates a diffeomorphism in a restricted restraint. A number of methods are used toward constrict region: 1) designed on behalf of every area inside the test MR picture, a limited investigate gap is used toward take out patches as of the preparation MR/CT pairs toward build MR as well as computed tomography dictionary; 2) k-Nearest Neighbors is utilized toward limit region inside the magnetic resonance vocabulary; 3 ) outlier recognition is performed toward limit region inside the computed tomography dictionary; 4) limited fasten Embedding is used toward resolving the magnetic resonance dictionary coefficients after representing the magnetic resonance difficult example. Below these restricted constraints, the coefficient weights are linearly transferred on or after magnetic resonance toward computed tomography also used to join the samples within the computed tomography dictionary toward make computed tomography calculations. The planned techniques have been evaluated on behalf of mind imagery under top of a dataset of 13 subjects. Every topic has T1- as well as T2-weighted magnetic resonance imagery, also a computed tomography picture through a sum of 39 imagery.

Reduction alteration is significant designed in favor of positron emission tomography rebuilding [14]. During PET/MR, magnetic resonance intensities are not straight correlated toward reduction coefficients that are desired inside positron emission tomography imaging. The reduction coefficient plan container be resulting in computed tomography imagery. Consequently, calculation of computed tomography substitute as of magnetic resonance imagery is preferred on behalf of reduction alteration inside positron emission tomography/magnetic resonance. Methods: This learning presents a patch-based technique used on behalf of computed tomography calculation beginning MR imagery; generate reduction maps used in favor of positron emission tomography rebuilding. Since no worldwide relation exists among magnetic resonance as 
well as computed tomography intensities, we suggest limited diffeomorphic mapping (LDM) used in favor of computed tomography calculation. During LDM, we suppose so as to MR as well as computed tomography patches are situated under top of two non-linear manifolds along with the mapping as of the magnetic resonance various toward the computed tomography various approximates a diffeomorphism in a limited restriction. Region is significant within LDM along with is controlled with means of the next techniques.

In addition, during the novel PET as well as magnetic resonance imaging scanner, simply magnetic resonance imagery are obtainable, which are alas not openly appropriate toward reduction alteration. These issues really prompt the expansion of methods used on behalf of dependable estimation of computed tomography [19] picture on or after its equivalent magnetic resonance picture of a similar topic. During this article, we suggest a learning-based technique to undertake this demanding difficulty. In particular, we initial separation a known magnetic resonance picture interested in a locate of patches. After that, meant on behalf of every patch, we utilize the prepared accidental jungle toward straight forecast a computed tomography patch since a prepared output, anywhere a novel collection model is as well used toward make sure the strong calculation.

An item detection scheme has been developed to utilize a novel group of limited picture features [16]. The features are invariant toward picture scaling, conversion, along with rotation, also incompletely invariant toward lighting change also affine otherwise 3D ridge These features divide alike properties through neurons inside lesser sequential cortex that are used in favor of object recognition inside mandrill image. Features are resourcefully detected during a theatrical filter advance so as to identify constant points within size space. Image keys are created toward permit on behalf of limited arithmetical deformations by means of representing unclear picture gradients inside numerous direction planes as well as on numerous scales. The keys are utilized because effort toward a nearest-neighbor indexing technique toward identifies applicant objects matches. The last confirmation of every competition is achieved through discovery a lowresidual least-squares result intended on behalf of the unidentified reproduction parameters.

The detection performance might be advance enhanced by means of adding novel SIFT characteristic types toward slot in color, texture, along with boundary grouping, also unreliable characteristic sizes also offsets. Scale-invariant boundary grouping toward create limited figure-ground discriminations [20-22] would exist mainly helpful on object limitations wherever environment disorder be able to interfere with extra features. The indexing with confirmation structure allows on behalf of each and every one type of level along with alternation invariant features toward survive included interested in a particular model illustration. Highest strength would be achieved through detecting a lot of dissimilar characteristic types as well as relying under top of the indexing as well as clustering toward choose individuals to facilitate are mainly helpful within an exacting picture.

\section{PROPOSED WORK}

The planned FMLND technique consists of three major stages: pre-processing of the magnetic resonance along with computed tomography imagery, learning of the local descriptors, as well as calculation of the predicted computed tomography picture via feature matching. The stages of the planned technique are exposed within Figure 1.

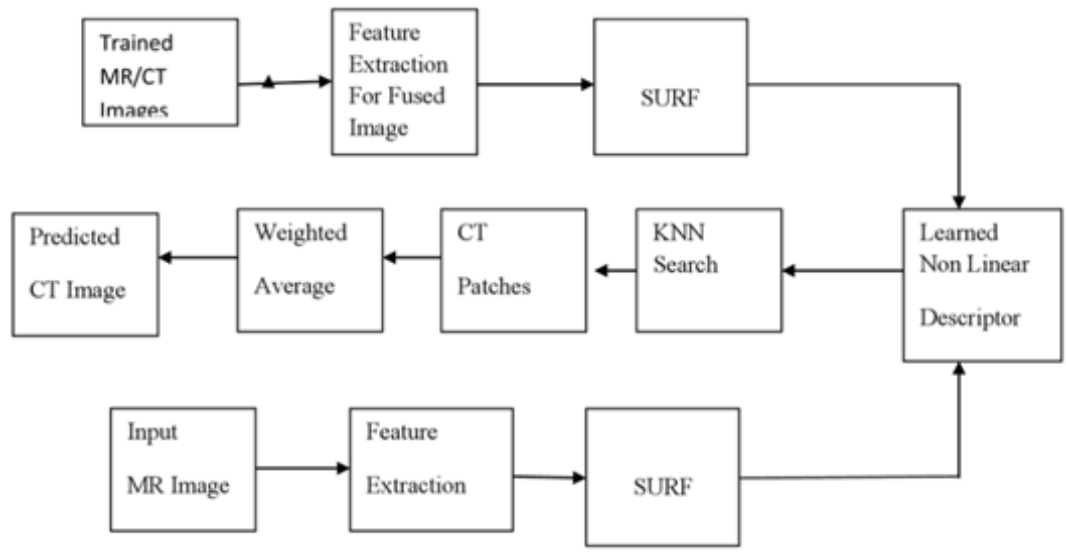

Figure 1. Proposed process of pCT synthesis from MR data through feature matching with learned nonlinear local descriptor

Int J Inf \& Commun Technol, Vol. 9, No. 1, April 2020: 46 - 56 


\subsection{Pre-processinbg of the MR and CT imagery}

The picture dataset utilized during this effort consists of T1w along with T2-weighted (T2w) magnetic resonance imagery, also the equivalent computed tomography imagery of 13 strong patients. The examiner parameter of the imagery are programmed within Table 1.

Toward begin through, the N-4 bias alteration algorithm ${ }^{1}[20]$ utilizes toward taking away the bias pasture artifact within the magnetic resonance imaging. After that, a strength normalization method [21] is useful toward decrease the difference diagonally the magnetic resonance imaging of dissimilar patients. The intensities of the magnetic resonance imagery are extended toward vary of $[0,100]$. The mind volumes (suitable imaging area) are divided on or after the CT scanning cot inside the CT imagery through thresholding. Lastly, spatial normalization is performed via linear affine listing (in FLIRT [22] within FSL2 by cross-correlation because the picture comparison assesses) toward line up the matching MR as well as computed tomography volumes of every tolerant. These linear affine registers serve because of the source of the following stages inside the planned technique.

Table 1. Scan Parameters of the dataset

\begin{tabular}{|c|c|}
\hline Dataset & Imaging parameters \\
\hline \multirow[t]{4}{*}{ MRI } & Scanner: GE Medical System Signa HDxt. \\
\hline & Magnetic field strength: 3T. \\
\hline & Slice thickness: $2.5 \mathrm{~mm}$ \\
\hline & In plane resolution: $0.47 \mathrm{~mm} \times 0.47 \mathrm{~mm}$ \\
\hline $\mathrm{T} 1_{\mathrm{w}}$ & $\mathrm{TR}=2884.74 \mathrm{~ms}, \mathrm{TE}=7.9 \mathrm{~ms}, \mathrm{TI}=960 \mathrm{~ms}$, flip angle $=90^{\circ}$. \\
\hline \multirow[t]{4}{*}{$\mathrm{T} 1_{\mathrm{w}}$} & $\mathrm{TR}=5000 \mathrm{~ms}, \mathrm{TE}=100.4 \mathrm{~ms}$, flip angle $=90^{\circ}$ \\
\hline & Scanner: GE Medical Systems Light Speed Pro 16. \\
\hline & Slice thickness: $2.5 \mathrm{~mm}$. \\
\hline & In-plane resolution: $0.47 \mathrm{mmx} 0.47 \mathrm{~mm}$. \\
\hline
\end{tabular}

Toward correctly forecast the predicted computed tomography imagery through means of a KNN estimator, the comparison otherwise space among the features must reproduce or else survive associated with the comparison otherwise space among the predicted targets. Usually, conservative magnetic resonance imaging (such as T1w as well as T2w) strength cannot reproduce the computed tomography assessment openly. In addition, the comparison among the raw patches of a magnetic resonance picture might poorly reproduce the comparison among the equivalent computed tomography patches. However, the hankie type's container exists recognized through the structural along with related information of huge spatial supports inside the magnetic resonance imaging. It is preferred to the limited descriptors of a magnetic resonance picture be able to signify the structural information also exist use toward classify efficient assessment used on behalf of KNN weakening. Supervised descriptor learning (SDL) techniques container is utilized to reach this target.

\subsection{Learning of the linear descriptors}

SURF's detectors also descriptor is not simply quicker, other than the past be too further repeatable as well as the last extra characteristic [23]. Hessian-based detectors are further constant as well as repeatable than their Harris based counterpart along with experimental toward approximation similar to the DoG container carry rate on a small rate during conditions of lost exactness [2] [6]. There are mainly two steps within SURF:

\subsubsection{Interest Point Detection}

SURF performance new picture toward integral picture. Integral picture which summed region tables is a midway illustration of the picture. It is the summation of intensity assessments of the entire pixels within contribution picture. Imagery rectangular area shaped via source $O=(0,0)$ along with one position $\mathrm{X}=(\mathrm{x}, \mathrm{y})$. It gives a quick calculation of box variety difficulty filters [2] [6].

$$
I_{\Sigma}(X)=\sum_{i=0}^{i \leq x} \sum_{j=0}^{j \leq y} I(i, j)
$$

Based on top of integral image, there are simply three operations (addition otherwise subtraction) necessitate on behalf of scheming calculation of intensity assessments of pixels more in the least vertical rectangular region. Integral image as shown in Figure 2. 


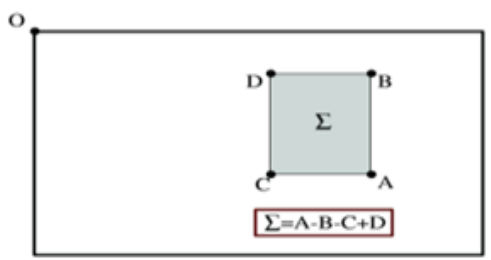

Figure 2. Using integral imagery, it takes only three additions also four memory accesses toward calculate the sum of intensities inside a rectangular region of any size

Integral picture is complicated through box filter. Box filter is estimated filter of Gaussian filter. The Hessian medium $\mathcal{H}(\mathrm{X}, \sigma)$, (since (2)) wherever $\mathrm{X}=(\mathrm{x}, \mathrm{y})$ of an picture $\mathrm{I}$, on level $\sigma$ is definite as:

$$
\mathcal{H}(X, \sigma)=\left[\begin{array}{ll}
L_{X X}(X, \sigma) & L_{X Y}(X, \sigma) \\
L_{X Y}(X, \sigma) & L_{Y Y}(X, \sigma)
\end{array}\right]
$$

Where $L_{X X}(X, \sigma)$ (Laplacian of Gaussian) be convolution of the Gaussian next arrange derived $\frac{\partial^{2}}{\partial X^{2}} g(\sigma)$ through (2) within position $X$ also likewise meant on behalf of $L_{X Y}(X, \sigma)$ along with $L_{Y Y}(X, \sigma)$.

\subsubsection{Interest Point Description}

Within order toward invariant toward image rotation, we recognize a reproducible direction used on behalf of the notice positions Because of that, initial compute Haar wavelet reactions within $\mathrm{x}$ along with $\mathrm{y}$ path inside a round area of radius 6s approximately the notice position, through scale $\mathrm{s}$ (sampling step depends on s) under which the notice position be detect can be seen at Figure 3. The dimension of wavelet which is weighing machine depended along with its side length is $4 \mathrm{~s}$. Toward calculate the answer within $\mathrm{x}$ or else y route on one size simply six operations are needed [24].

Previously the wavelet reactions are designed as well as weighted through a Gaussian $\sigma=2 \mathrm{~s}$ centered on the notice position. The reactions are representing since tips within a gap through the straight reaction force next toward the abscissa as well as the perpendicular reaction force beside the ordinate. Find maximum the amount of every response which is wavelet reaction within every down transom $(\pi / 3$ transom direction) (observe Figure 4). The flat, as well as upright responses inside the window, are summed. From these two horizontals as well as vertical, summed responses next give way a limited direction vector. The direction of the notice position container exists definite through result best such vector more than every transom.
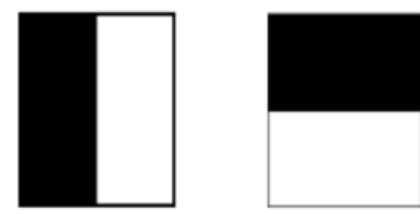

Figure 3. Haar wavelet filters toward calculate hr response within $\mathrm{x}$ (left) as well as y direction (right).

The shady parts contain the weight -1 along with the light parts +1

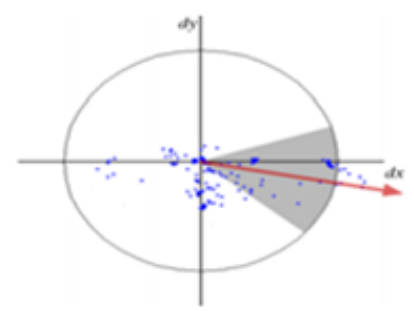

Figure 4. Orientation assignment: A Sliding orientation windoe of size $\pi / 3$ detects the dominant orientation of the Gaussin weighted Haar wavelet responses at every sample point within a circular neighborhood around the interest point. 
Toward extract, the descriptor, cube area which dimension is 20 s are constructed under top of interested points. Examples of such square regions are illustrated in Figure 5.

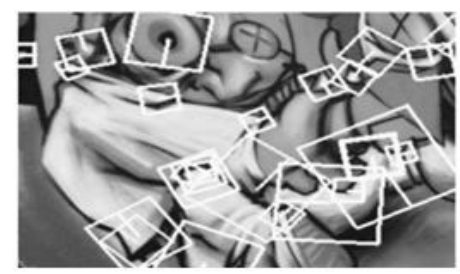

Figure 5. Detail of the Graffiti scene showing the size of the oriented descriptor window at different

The wavelet responses $d_{x}$ with $d_{y}$ are summed up more than every sub-region along with form a first set of entries inside the feature vector. During order toward bring in information concerning the polarity of the intensity changes, extract the sum of the absolute assessments of the responses, $\left|d_{x}\right|$ along with $\left|d_{y}\right|$, each sub-region have a four-dimensional descriptor vector $\mathrm{V}$ used for its underlying intensity structure $\mathrm{V}=\left(\sum \mathrm{d}_{\mathrm{x}}\right.$, $\left.\sum \mathrm{d}_{\mathrm{y}}, \sum\left|\mathrm{d}_{\mathrm{x}}\right|, \sum\left|\mathrm{d}_{\mathrm{x}}\right|\right)$. Concatenating this used for all, $4 \mathrm{x} 4$ sub-regions, with these results within a descriptor vector of length is 64 . The wavelet responses are invariant toward a bias inside illumination (offset) as well as Invariance toward contrast (a scale factor) is achieved through turning the descriptor into a unit vector.

\subsection{Learning of the nonlinear descriptors}

The learned descriptor (linear descriptor) within (3) is presently the linear embedding of the most important descriptor. Toward get further dominant descriptor; further expand the linear formulation within. (1) As well as (3) interested in the non-linear formulations by the kernel method. The main descriptor is predictable interested in a high-dimensional gap through a non-linear map $\Phi(d)$. The characteristic plan $\Phi(d)$ of the positive definite (PD) essential part $K\left(d, d^{\prime}\right)[25]$ maps the linear descriptor $d$ interested in the Hilbert gap [26] through linear internal creation <. >, i.e.

$$
K\left(d, d^{\prime}\right)=\Phi(d)^{T} \Phi\left(d^{\prime}\right)
$$

Toward reduce in the direction of bare-bones optimization difficulty; we take under the open characteristic plan technique toward estimated a known positive definite kernel $K\left(d, d^{\prime}\right)$ through a characteristic map $\Phi(d): \mathrm{d} \longrightarrow R^{q}$. The attained protuberance container is approximated through linear enhanced supervised descriptor learning technique. Precise characteristic maps be appropriate below the restraint to just if a known non-Euclidean metric be preservative along with standardized. The preservative kernel $K\left(d, d^{\prime}\right)$ on top of $R^{q}$ container is expressed as:

$$
K\left(d, d^{\prime}\right)=\sum_{j=1}^{q} K\left(d_{j}, d_{j}^{\prime}\right)
$$

The standardized kernel container moreover be expressed since $\lambda K\left(d, d^{\prime}\right)=K\left(\lambda d, \lambda d^{\prime}\right)$ meant on behalf of every $\lambda>0$. Frequently used kernel [27-29] comprises the subsequent: $\chi^{2}$ kernel:

$$
K\left(d, d^{\prime}\right)=2 d d^{\prime} /\left(d+d^{\prime}\right)
$$

Jensen-Shannon (JS) kernel:

$$
K\left(d, d^{\prime}\right)=(d / 2) \log _{2}\left(d+d^{\prime}\right) / d+\left(d^{\prime} / 2\right) \log _{2}\left(d+d^{\prime}\right) / d^{\prime}
$$

Intersection kernel:

$$
K\left(d, d^{\prime}\right)=\min \left\{d, d^{\prime}\right\}
$$

The open feature map method approximates protuberance $\Phi(d)$ by separate Fourier change through a sample rate $r$ while $\widehat{\Phi}(d)$. Mainly thick SURF descriptors are predictable non-linearly interested in a high 
dimensional gap $R^{(2 r+1) q \times 1}$ because the open characteristic map $\widehat{\Phi}(d)$. Every predictable main dense SURF descriptor is next rearranged interested in an $\mathrm{M} \times \mathrm{N}$ matrix as well as joint through the raw patch since a nonlinear descriptor. Analogously toward control learn of linear descriptor, we utilize the similar formulation within (1) toward discover the invariant medium $\mathbf{W}$ along with the discriminative medium $\mathbf{V}$ used on behalf of the nonlinear high-dimensional descriptor $\widehat{\Phi}(d)$. A final LND is obtained as $d^{\prime}=W^{T} \widehat{\Phi}(d) V$.

Figure 6. (a), as well as (b) here, signify complete fault of medium $\mathbf{W}$ along with $\mathbf{V}$ consequences used on behalf of Equation (1). The dissimilarity within perpendicular alignment is indicating complete fault among the present as well as before the solution through the iterations. Matrices $\mathbf{W}$, as well as V, remains unaffected following the third iteration, therefore representing the best explanation of Eq. (1) have converged. Toward undoubtedly clarify the thought of learned non-linear descriptor, we supply the pseudocode used in favor of the learning process within procedure 1.

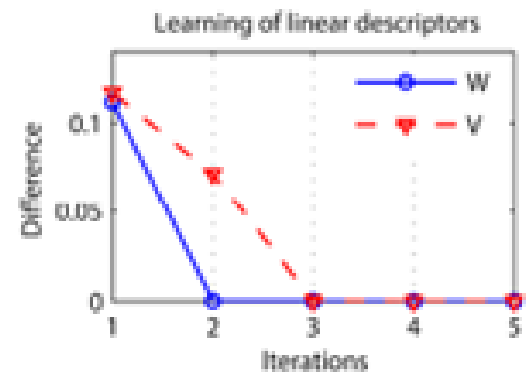

(a)

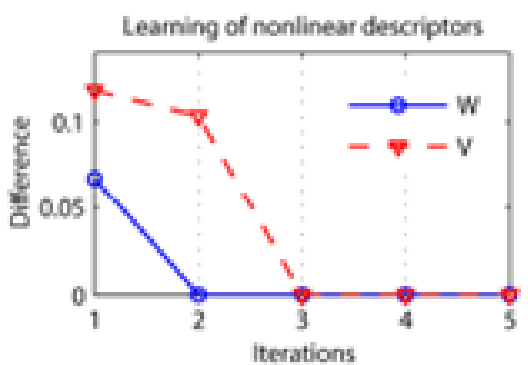

(b)

Figure 6. Mean absolute error of discriminative matrix $\mathrm{W}$ along with $\mathrm{V}$ among the present outcome also the previous result. a) Result of linear descriptors. b) Result of nonlinear descriptors.

\section{Algorithm 1: Learning of the nonlinear local descriptors}

Input: Preparation pair of the magnetic resonance along with computed resonance imagery.

Output: Discriminative medium W along with V.

Stage-1: Assume intense SURF toward taking out the extensive variety of features as well as the structural information of the MR imagery.

Stage-2: Plan the extract intense SURF descriptors interested in the high dimensional space by open feature maps, along with subsequently they obtain descriptors included through raw patch strength indicate the nonlinear descriptors.

Stage-3: Gather a bulky amount of MRI nonlinear descriptor patches along with their equivalent CT strength patches.

Stage-4: Explore C adjacent neighbors of every CT area inside a constriction spatial variety toward attain the medium $\mathrm{S}$.

Stage-5: Iteratively explain the best role Equation (1) to discover the best effect also find the discriminative matrices $\mathrm{W}$ along with $\mathrm{V}$.

\subsubsection{Calculation of the predicted computed tomography picture through feature matching}

Designed on behalf of every position $\mathrm{x} \subset \mathrm{C}$ inside input $\mathrm{T} 1 \mathrm{w}$ as well as $\mathrm{T} 2 \mathrm{w}$ magnetic resonance Imagery, reduce the rate purpose toward approximation the equivalent computed tomography area $f(x)$ centered on $x$ :

$$
J=\sum_{x \subset c}^{c} \| f(x)-\hat{f}(x)_{2}^{2}
$$

Where $\hat{f}(x)$ an estimator along with $\mathrm{C}$ be the voxel locate inside suitable picture area. K-Nearest Neighbor weakening is utilizing toward guess the assessment of purpose $\hat{f}(x) . k$ nearest neighbors be search as well as chosen in a permanent spatial variety of every position $\mathrm{x}$ within the magnetic resonance imagery used on behalf of the learn linear otherwise non-linear descriptor $d_{x}$. The $k$ nearest neighbors of $d_{x}$ within Magnetic resonance imagery along with equivalent computed tomography areas container is denoted as $\left\{D_{i}^{M R}, D_{i}^{C T}(i=1,2, \ldots k)\right\}$. During the diverse of the magnetic resonance descriptors, a linear estimate $\mathrm{d}_{\mathrm{x}}$ container is obtained by: 


$$
\underset{w}{\arg \min }\left\|d_{x}-D_{k}^{M R} w\right\|_{2}^{2}, \text { s.t. }, \sum_{i=1}^{k} w_{i}=1
$$

The biased coefficient vector $w$ be utilized toward calculating the quantity of assessment among descriptor $\mathrm{d}_{\mathrm{x}}$ with its $k$ adjacent neighbors. The biased coefficient vector $\mathbf{w}$ container is considered basically by a Gaussian kernel function:

$$
w_{i}=\exp \left(-\left\|d_{x}-D_{i}^{M R}\right\|_{2}^{2}\right) / \sum_{j=1}^{k} \exp \left(-\left\|d_{x}-D_{j}^{M R}\right\|_{2}^{2} /\left(2 \sigma^{2}\right)\right)
$$

$\sigma$ is determined by the following:

$$
\sigma=\left\|d_{x}-d_{x}^{(q)}\right\|_{2},
$$

Where $d_{x}^{(q)}$ is the $\mathrm{q}^{\text {th }}$ nearest neighbor of $d_{x}$. During the experiments, parameter $\sigma$ usually workings fine when $\mathrm{q}=4$. Once obtain the weighted coefficients $\boldsymbol{w}$, the CT vector or else area used on behalf of every MR descriptor $d_{x}$ container are estimated as:

$$
f^{\prime}(x)=D_{k}^{C T} w,
$$

With the equivalent, CT patches DCT $k$ along with the weighted coefficient vector $w$. Following every one of the CT patches used on behalf of an input MR picture is predictable, a biased typical process is performed under top of the overlapped CT patches toward get the last predict pCT picture.

\subsection{Simulation of $\mathrm{AC}$}

Usually, together with the magnetic resonance, as well as CT imagery used on behalf of one patient, is simply acquired within the health center. Though, MRI, CT also PET imagery meant in favor of single patient is hardly ever every obtainable. Hofmann et al. [4] planned to make use of a replicated usual PET picture to estimate the collision of pCT attenuation correction during the evaluation of accurate computed tomography attenuation correction. Subsequent the advance used, utilize the Positron emission tomography/Magnetic Resonance Imaging (MNI $152 \mathrm{~T} 1 \mathrm{w}$ ) template3 toward reproduce the lost PET records toward assessing the presentation of attenuation correction[22]. We initial line up the magnetic resonance imaging pattern toward the magnetic resonance imaging of every issue patient by the deformable register toolbox FNIRT ${ }^{4}$ within the $\mathrm{FSL}^{2}$. The obtained transformations are next useful toward the ${ }^{18} \mathrm{~F}-\mathrm{FDG}$ PET template picture toward get the simulated PET imagery.

Within [7], the attenuation assessments were transformed like of Hounsfield units (HU) toward LACs by $511 \mathrm{keV}$ within $\mathrm{cm}^{-1}$. Reduction map ( $\mu$-map) is computed via using a piece-wise linear map [30] conversion; the computed tomography assessments be transformed interested in LACs like follow:

$$
\mu^{P E T}\left(I^{C T}\right)= \begin{cases}e \times\left(I^{C T}+1000\right) & I^{C T} \leq 0 H U \\ e \times 1000+a \times I^{C T} & I^{C T}>0 H U\end{cases}
$$

Where $I^{C T}$ denote the CT picture indicator strength, e=9.6 $\times 10-5 \mathrm{~cm}^{-1}$, as well as $a$ is a stable depending under X-ray tube voltages.

The $\mu$-map resulting start the accurate computed tomography as well as $\mathrm{pCT}$ is primary predictable toward obtains the reduction records of PET $_{\mathrm{pCT}}$ by the Radon along with converse Radon transformations. Toward get $\mathrm{PET}_{\mathrm{pCT}}$, the uncorrected sonogram records used for attenuation are required:

$$
\operatorname{Sin}=R a(P E T) \times \exp \left[-R a\left(\mu^{P E T}(C T)\right)\right]
$$

Where Ra denote Radon transform. Next, $\mathrm{PET}_{\mathrm{pCT}}$ is reconstructed by the corrected sonogram once Sin is obtained:

$$
P E T_{p C T}=R a^{-1}\left(\operatorname{Sin} \times \exp \left[R a\left(\mu^{P E T}(p C T)\right)\right]\right)
$$

wherever $\mathrm{Ra}^{-1}$ be the converse Radon transforms. 


\subsection{Evaluation measures}

We estimate the method used in favor of predict pCT imagery with case-wise leave-one-out crossvalidation (LOOCV). Subsequent Ladefoged et al. [31], four quantitative procedures be working toward estimate the presentation of the techniques.

- Mean absolute error (MAE): Mean absolute error procedures the voxel-wise error (within HU), which container exist formulate since follow:

$$
M A E=\frac{|C T-p C T|}{C}
$$

Wherever $\mathrm{C}$ be the voxel locate within a suitable picture area, also computed tomography along with predicted computed tomography indicate the accurate CT along with the predict pCT picture, correspondingly.

- Peak signal-to-noise ratio (PSNR): Peak signal to noise ratio (within $\mathrm{dB}$ ) is defined like follow:

$$
P S N R=10 \log _{10}\left[\frac{Q^{2}}{\|C T-p C T\|_{2}^{2} / C}\right]
$$

Where Q denote the highest assessment of intensity within the properly computed tomography as well as pCT.

\section{EXPERIMENTAL RESULTS}

The result of the experiment is represented in Figure 7 and predicted CT image from MRI as shown in Figure 8. The comparission result of SIFT and SURF as shown in Table 2.

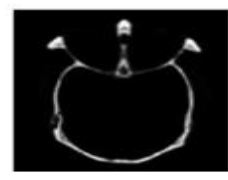

a

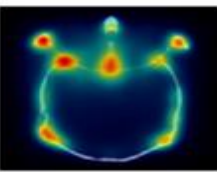

$\mathbf{g}$

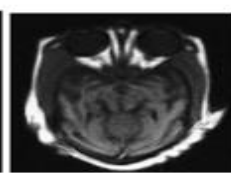

b

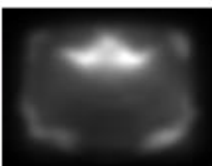

$\mathbf{h}$

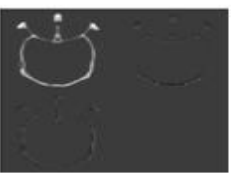

c

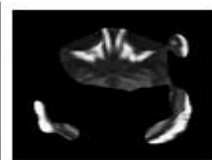

i

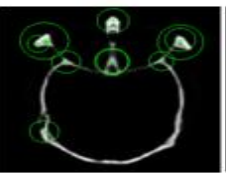

d

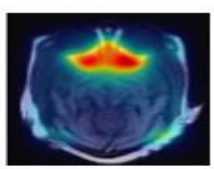

j

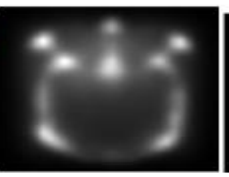

e

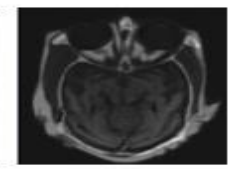

$\mathbf{k}$

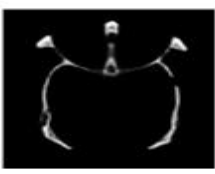

f

Figure 7. (a) CT Image, (b) MRI Image, (c) CT Calculation, (d) SURF features, (e) \& (h) High Dimensional Space, (f) \& (i) Corresponding LNDs, (g) \& (j) SDL and (k) KNN Regression

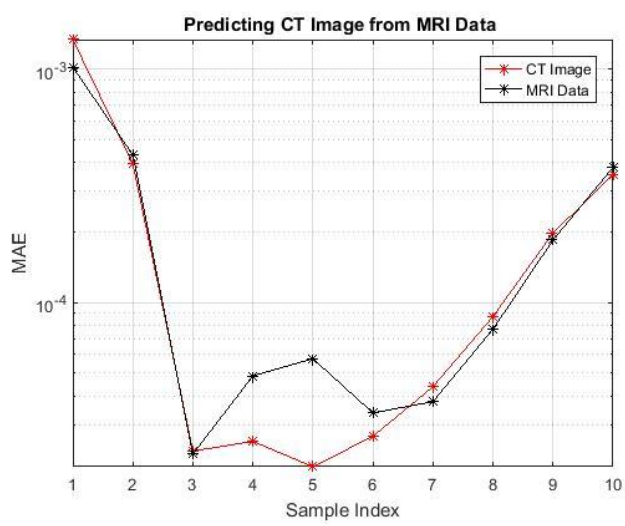

Figure 8. Predicted pCT imagery 
Table 2. Comparison of SIFT and SURF by Using PSNR and MAE

\begin{tabular}{ccc}
\hline Methods/Parameters & PSNR & MAE \\
\hline SIFT & 27.5021 & 80.9579 \\
SURF & 34.3776 & 89.8036 \\
\hline
\end{tabular}

\section{CONCLUSION}

During this assessment, we suggest a constituent coordinate method by means of learned nearby descriptors used for anticipating CT on or after MR picture information. The necessary descriptors of the magnetic resonance picture are primary anticipated toward a high dimensional gap toward get non-linear descriptors utilizing an open component drawing. These descriptors are higher through the implementation of a better SDL computation. The experiment results illustrate to the learned nonlinear descriptors are successful used for thick coordinating along with pCT expectation. In addition, the planned CT estimate approach can accomplish focused execution contrasted along with a few cutting-edge techniques.

\section{FUTURE WORK}

During outlook study, our effort resolves live extensive toward deep learning, also medical picture retrieval resolve be further through collect a lot of subjects used in favor of medical application. The medical purpose designed in favor of MRI-based current RT conduct preparation resolve also investigates carefully.

\section{REFERENCES}

[1] Zaidi, M.- L. Montandon, and D. O. Slosman, "Attractive reverberation imaging-guided lessening and disperse remedies in three-dimensional mind positron outflow tomography," Medical Physics, vol. 30, no. 5, pp. 937-948, 2003.

[2] S.- H. Hsu, Y. Cao, K. Huang, M. Feng, and J. M. Balter, "Examination of a strategy for creating engineered CT models from MRI outputs of the head and neck for radiation treatment," Physics in prescription and science, vol. 58, no. 23, pp. 8419-8435, 2013.

[3] D. Izquierdogarcia, A. E. Hansen, S. Förster, D. Benoit, S. Schachoff, S. Fürst, K. T. Chen, D. B. Conde, and C. Catana, "An SPM8-based Advance for Attenuation Correction Combining Segmentation and Non-unbending Template Formation: Application to Simultaneous PET/MR Brain Imaging," Journal of Nuclear Medicine, vol. 55, no. 11 , pp. 1825-30, 2014

[4] M. Hofmann, F. Steinke, V. Scheel, G. Charpiat, J. Farquhar, P. Aschoff, M. Brady, B. Schölkopf, and B. J. Pichler, "X-ray Based Attenuation Correction for PET/MRI: A Novel Advance Combining Pattern Recognition and Atlas Registration," Journal of Nuclear Medicine, vol. 49, no. 11, pp. 1875-1883, 2008.

[5] N. Burgos, M. J. Cardoso, K. Thielemans, M. Modat, S. Pedemonte, J. Dickson, A. Barnes, R. Ahmed, C. J. Mahoney, J. M. Schott, J. S. Duncan, D. Atkinson, S. R. Arridge, B. F. Hutton, and S. Ourselin, "Constriction Correction Synthesis for Hybrid PET-MR Scanners: Application to Brain Studies," IEEE Transactions on Medical Imaging, vol. 33, no. 12, pp. 2332-2341, 2014.

[6] I. Mérida, N. Costes, R. A. Heckmann, A. Drzezga, S. Förster, and A. Mallets, "Assessment of a few multi-map book strategies for PSEUDO-CT age in cerebrum MRI-PET weakening rectification," IEEE Twelfth International Symposium on Biomedical Imaging, pp. 1431-1434, 2015.

[7] V. Keereman, Y. Fierens, T. Broux, Y. De Deene, M. Lonneux, and S. Vandenberghe, "X-ray Based Attenuation Correction for PET/MRI Using Ultrashort Echo Time Sequences," Journal of Nuclear Medicine, vol. 51, no. 5, pp. $812-818,2010$.

[8] A. Johansson, M. Karlsson, and T. Nyholm, "CT substitute got from MRI groupings with ultra-short reverberation time," Medical Physics, vol. 38, no. 5, pp. 2708-2714, 2011.

[9] M. E. Jens, M. K. Hans, L. Koen Van, H. H. Rasmus, A. L. A. Jon, and A. Daniel, "A voxel-based examination for MRI-just radiotherapy of the mind utilizing ultrashort reverberation times," Physics in Medicine and Biology, vol. 59, no. 23, pp. 7501-7519, 2014.

[10] S. Roy, W.- T. Wang, A. Carass, J. L. Ruler, J. A. Butman, and D. L. Pham, "PET Attenuation Correction Using Synthetic CT from Ultrashort Echo-Time MR Imaging," Journal of Nuclear Medicine, vol. 55, no. 12, pp. 2071-2077, 2014.

[11] M. R. Juttukonda, B. G. Mersereau, Y. Chen, Y. Su, B. G. Rubin, T. L. S. Benzinger, D. S. Lalush, and H. A, "MRbased weakening amendment for PET/MRI neurological examinations with consistent esteemed constriction coefficients for bone through a change from R2* to CT-Hounsfield units," NeuroImage, vol. 112, pp. 160-168, 2015. 
[12] G. Delso, F. Wiesinger, L. I. Sacolick, S. S. Kaushik, D. D. Shanbhag, M. Hüllner, and P. Veit-Haibach, "Clinical Evaluation of Zero-Echo-Time MR Imaging for the Segmentation of the Skull," Journal of Nuclear Medicine, vol. 56, no. 3, pp. 417-422, 2015.

[13] Y. Wu, W. Yang, L. Lu, Z. Lu, L. Zhong, R. Yang, M. Huang, Y. Feng, W. Chen, and Q. Feng, "Forecast of CT Substitutes from MR Imagery Based on Local Sparse Correspondence Combination," Medical Image Computing and Computer-Assisted Intervention - MICCAI, pp. 93-100, 2015.

[14] Y. Wu, W. Yang, L. Lu, Z. Lu, L. Zhong, M. Huang, Y. Feng, Q. Feng, and W. Chen, "Forecast of CT Substitutes from MR Imagery Based on Local Diffeomorphic Mapping for Brain PET Attenuation Correction," Journal of Nuclear Medicine, vol. 57, no. 10, pp. 1635-1641, 2016.

[15] T. Huynh, Y. Gao, J. Kang, L. Wang, P. Zhang, J. Lian, and D. Shen, "Assessing CT Image from MRI Data Using Structured Random Forest and Auto-Context Model," IEEE Transactions on Medical Imaging, vol. 35, no. 1, pp. 174-183, 2016.

[16] D. G. Lowe, "Article acknowledgment from nearby scale-invariant highlights," The Proceedings of the Seventh IEEE International Conference on Computer Vision, vol. 2, pp. 1150-1157, 1999.

[17] A. Vedaldi, and A. Zisserman, "Proficient Additive Kernels by means of Explicit Feature Maps," IEEE Transactions on Pattern Analysis and Machine Intelligence, vol. 34, no. 3, pp. 480-492, 2012.

[18] L. Zhong, L. Lin, Z. Lu, Y. Wu, Z. Lu, M. Huang, W. Yang, and Q. Feng, "Foresee CT picture from MRI information utilizing KNN-relapse with scholarly neighborhood descriptors," IEEE Thirteenth International Symposium on Biomedical Imaging, pp. 743-746, 2016.

[19] Shruti Bhargava, "A Study on Various Speckle Noise Diminution Techniques," International Journal of Recent Development in Engineering and Technology, vol. 6, no. 2, 2017

[20] Shruti bhargava, Ajay Somkuwar, "Denoising Mri Images Using Wavelet-Based Bivariate Shrinkage Method," CCET Journal of Science \& Engineering Education, vol. 1, pp. 55-61, 2016

[21] Shruti Bhargava and Ajay Somkuwar, "Estimation of Noise Removal Techniques in Medical Imaging Data-A Review," Journal of Medical Imaging and Health Informatics, vol. 6, 1-10, 2016.

[22] Shruti Bhargava and S.P.V. Subbarao, "Evaluation of Different Noise Removal Filterization Techniques in Imaging”, International Journal of Pure and Applied Mathematics, vol. 117, no. 21, pp. 605-612, 2017.

[23] N. S. Altman, "An Introduction to Kernel and Nearest-Neighbor Nonparametric Regression," The American Statistician, vol. 46, no. 3, pp. 175-185, 1992.

[24] N. J. Tustison, B. B. Avants, P. A. Cook, Y. Zheng, A. Egan, P. A. Yushkevich, and J. C. Hmm," N4ITK: Improved N3 Bias Correction," IEEE Transactions on Medical Imaging, vol. 29, no. 6, pp. 1310-1320, 2010.

[25] L. G. Nyul, J. K. Udupa, and Z. Xuan, "New variations of a strategy for MRI scale institutionalization," IEEE Transactions on Medical Imaging, vol. 19, no. 2, pp. 143-150, 2000.

[26] Shruti Bhargava, Ajay Somkuwar "Evaluation of Noise Exclusion of Medical Images Using Hybridization of Particle Swarm Optimization and Bivariate Shrinkage Methods," International Journal of Electrical and Computer Engineering (IJECE), vol. 5, no. 3, pp. 421-428, 2015.

[27] M. Jenkinson, P. Balustrade, M. Brady, and S. Smith, "Improved Optimization for the Robust and Accurate Linear Registration and Motion Correction of Brain Imagery," NeuroImage, vol. 17, no. 2, pp. 825-841, 2002.

[28] Z. Xiantong, W. Zhijie, Y. Mengyang, and L. Shuo, "Administered descriptor learning for multi-yield relapse," IEEE Conference on Computer Vision and Pattern Recognition, pp. 1211-1218, 2015.

[29] Z. Zhang, and K. Zhao, "Low-Rank Matrix Approximation with Manifold Regularization," IEEE Transactions on Pattern Analysis and Machine Intelligence, vol. 35, no. 7, pp. 1717-29, 2013.

[30] B. Scholkopf, and A. J. Smola, "Learning with Kernels: Support Vector Machines, Regularization, Optimization, and Beyond," IEEE Transactions on Neural Networks, pp. 781-781, 2005.

[31] M. Hein, O. Bousquet, Z. Ghahramani, R. Cowell, Cowell, and Ghahramani, "Hilbertian measurements and positive distinct parts on likelihood measures," Proceedings of Aistats, no. 2005, pp. 136-143, 2005. 